\section{Microcapillary Reactors Using Solid-Phase DNA Sequencing for Direct Sample Introduction into Slab Gels}

BioTechniques 28:904-912 (May 2000)

\begin{abstract}
Solid-phase microreactors have been prepared in glass capillaries for DNA sequencing applications using slab gel electrophoresis, which consisted of a fused silica capillary (i.d. $=100 \mu \mathrm{m} ;$ o.d. $=365 \mu \mathrm{m}$; length $=15 \mathrm{~cm}$; volume $=1.2 \mu \mathrm{L}$ ) that contained a covalently bound biotin molecule. With the addition of streptavidin to the capillary, an anchoring site was produced for the tethering of biotinylated DNA sequencing templates to the wall of the capillary. Using a four-lane, single dye primer chemistry sequencing strategy, the individual tracts were prepared in the capillaries using cycle sequencing (20 thermal cycles) on a $P C R$-generated $\lambda$-bacteriophage template (about $1000 \mathrm{bp}$ ). The dye label in this case was a fluorescent tag that displayed emission properties in the near-IR and could be processed on an automated sequencer. The read length was found to be 589 bases, which was determined primarily by the fractionating power of the gel. It was also found that the tethering system was very stable to typical cycle sequencing conditions, with the amount of tethered DNA lost amounting to $40 \%$ after 120 thermal cycles. The ability to use dye terminator chemistry was also investigated by using a near-IR dye-labeled terminator (ddGTP). It was found that the quality of the ladder that was generated was comparable to that obtained in a conventional sample preparation format. However, ethanol precipitation was required before gel loading to remove excess terminator.
\end{abstract}

\section{INTRODUCTION}

Solid-phase DNA sequencing strategies have developed into important processes for high-throughput applications. The important attributes associat- ed with solid-phase approaches include: $(i)$ the generation of high-purity, single-stranded products. The solid support can act as a capture medium for the double-stranded template generated using PCR, and once captured, the complement can be effectively removed using heat or $\mathrm{NaOH}$ melting. In fact, elution of the complement can allow for bidirectional sequencing of one PCR product (5); (ii) the removal of interferences produced from the PCR or sequencing reactions. The removal of excess primers, salts or dNTPs can easily be accomplished by capture of the sequencing template on the solid support with no need for centrifugation or precipitation steps. Many times the removal of these interferences can improve banding in the electrophoresis step of the analysis, especially in capillary gel electrophoresis (19); (iii) solidphase sequencing is conducive to automation. Fluid handling is greatly simplified in most solid-phase approaches, and many of the steps can simply be handled by conventional robotic workstations $(12,21)$.

The common support medium used in many solid-phase approaches is magnetic beads $(3,6,8,9,18,20,22)$ or ultra-small polystyrene beads (4) coated with streptavidin. By producing a PCR product using a primer containing a biotin molecule on the $5^{\prime}$ end, the PCR product can be tethered to the bead with magnetic capture to isolate the template for further processing. An alternative method is to use streptavidin agarose gels to immobilize the DNAs $(7,17)$.

The detection of the DNA sequencing ladders that were produced using solid-phase processing can easily be accomplished using fluorescence $(1,11$, 15). In most sequencing applications that use solid-phase approaches, dyelabeled primers are used. However, Schofield and co-workers have shown that dye terminator chemistry can also be used in solid-phase sequencing strategies (14), which permits generating the four terminated products in one reaction vessel and minimizes postsample handling before gel loading.

Recently, we extended the solidphase approach to microcapillary tubes, which offers the additional advantage of being able to scale the reaction to nanoliter volumes with a concomitant savings arising from the reduction in consumables during DNA polymerization (16). In addition, microcapillaries are easily manipulated with robotics for automated operation, and fluid handling can be accomplished using electrokinetic pumping. In our work, the microcapillary tube was functionalized with biotin to serve as the anchoring scaffold for the DNA template (Figure 1, A and B). This was followed by incubation of the tube with streptavidin and then using another binding site of the tetrameric streptavidin protein to attach the PCR product containing a biotin handle to the wall of the microcapillary tube (biotin:streptavidin:biotin layer). The advantage of tethering the biotin directly to the wall of the capillary, and not to streptavidin, is that one can simply strip the DNA from the reactor using heat and formamide without removing the biotin layer, allowing regeneration of the reactor. Using this format, we have demonstrated that cycle sequencing using Taq DNA polymerase (Amersham Pharmacia Biotech, Piscataway, NJ, USA) and a single dye tract can be accomplished in a volume of about $64 \mathrm{~nL}$.

Here, we demonstrate the use of this microcapillary solid-phase DNA sequencing reactor interfaced with conventional slab gel electrophoresis. Since the slab gel format can accept larger volumes of materials as compared to capillary gel electrophoresis (sample volume approximately $10 \mathrm{~nL}$ for capillary gel electrophoresis and sample volume approximately $5 \mu \mathrm{L}$ for slab gel electrophoresis), the reactor volume was scaled-up by simply increasing the length of the capillary as well as its internal diameter. Following generation of the sequencing ladders, the tube could be heated to denature the DNAs. The entire contents of the capillary reactor were then pressure injected into a well of the slab gel for fractionation of the sequencing products. In addition, we show that the anchoring system is stable toward typical cyclesequencing conditions and also can be subjected to multiple sequencing rounds. Finally, data are included to demonstrate the feasibility of generating sequencing products using near-IR fluorescent dye terminator chemistry. 


\section{MATERIALS AND METHODS}

\section{Generation of Biotinylated PCR Product}

A 1-kb target was amplified by PCR from bacteriophage $\lambda$-DNA in a PerkinElmer 2400 series thermocylcer (PE Biosystems, Foster City, CA, USA). The PCR primers were designed (Figure 1C) to amplify bases 6610-7630 of the $\lambda$-DNA target. The PCR mixture contained $1 \mu \mathrm{L} \lambda$-DNA (5 pmol), $10 \mu \mathrm{L}$ $10 \times$ PCR buffer $[20 \mathrm{mM}$ Tris- $\mathrm{HCl}(\mathrm{pH}$ 8.3), $50 \mathrm{mM} \mathrm{KCl}$ and $15 \mathrm{mM} \mathrm{MgCl}_{2}$ ], 5 $\mu \mathrm{L} 24$-base biotinylated forward primer $(10 \mu \mathrm{M}), 5 \mu \mathrm{L} 24$-base reverse primer $(10 \mu \mathrm{M}), 0.5 \mu \mathrm{L}$ AmpliTaq ${ }^{\circledR}$ DNA polymerase (PE Biosystems) and 70.5 $\mu \mathrm{L}$ double-distilled water. Thirty-five PCR cycles were performed using the following temperature program: (i) denature dsDNA at $94^{\circ} \mathrm{C}$ for $45 \mathrm{~s}$; (ii) anneal primers to template at $66^{\circ} \mathrm{C}$ for 30 s; and (iii) extend primers at $72^{\circ} \mathrm{C}$ for 90 s. Following PCR amplification, the biotinylated PCR products were purified using Amicon ${ }^{\circledR}$ Microcon ${ }^{\circledR}$ concentrators (Millipore, Bedford, MA, USA) with molecular cutoffs at $50 \mathrm{kDa}$. The PCR product was stored in water at $-20^{\circ} \mathrm{C}$ until needed.

\section{Immobilization of Biotinylated PCR Product to Capillary Wall}

The PCR product was immobilized to the wall of a fused silica capillary using a biotin:streptavidin:biotin system (16), which is briefly outlined here. Fused silica capillary tubes $(100 \mu \mathrm{m}$ i.d.; $365 \mu \mathrm{m}$ o.d.) were cut into $100-\mathrm{cm}$ sections and rinsed successively with 1 $\mathrm{M} \mathrm{NaOH}$, double-distilled water and 1 $\mathrm{M} \mathrm{HCl}$ for $30 \mathrm{~min}$ each. This was followed by purging the capillary with air for $10 \mathrm{~min}$ and finally oven-baking the capillary at $200^{\circ} \mathrm{C}$ for several hours. Af- ter drying, the capillary was filled with a solution of 5\% (3-aminopropyl)triethoxysilane (Sigma, St. Louis, MO, USA) in acetone. The capillary was allowed to stand for $30 \mathrm{~min}$ and then purged with air for $3 \mathrm{~min}$ and finally incubated at $45^{\circ} \mathrm{C}$ for about $24 \mathrm{~h}$. Next, the capillary was filled with a bicarbonate solution $(50 \mathrm{mM}$; $\mathrm{pH} 8.3)$ containing $5 \mathrm{mg} / \mathrm{mL}$ NHS-LC-biotin (Sigma) for $4 \mathrm{~h}$ at room temperature. Following this treatment, the capillary was briefly rinsed with double-distilled water and gravity-filled with a $4 \mathrm{mg} / \mathrm{mL}$ solution of streptavidin (Sigma) that was prepared in $50 \mathrm{mM}$ phosphate buffer $(\mathrm{pH}$ 7.4) at $4^{\circ} \mathrm{C}$. Finally, the streptavidincoated capillary was rinsed with water and treated by gravity flow, which was generated by adjusting the height difference between the two ends of the capillary, with the biotinylated PCR product at $4^{\circ} \mathrm{C}$. Excess PCR product was removed by a final water wash. 


\section{Short Technical Reports}

Near-IR Fluorescence Sequencing with Dye-Labeled Primer

Extension of the immobilized PCR product was accomplished directly within a $15-\mathrm{cm}$ section of microcapillary (total volume about $1.2 \mu \mathrm{L}$ ). Since the original PCR product was immobilized as a duplex, it was thermally denatured at $95^{\circ} \mathrm{C}$ for $5 \mathrm{~min}$ with constant gravity flushing to remove the complement. For dye-primer chemistry, four reactors were used, one for each base. Each solid-phase capillary reactor was filled with the appropriate extension mixture, which consisted of $1 \mu \mathrm{L} 21-\mathrm{bp}$ IRD-800 labeled sequencing primer (LI-COR, Lincoln, NE, USA); $5 \mu \mathrm{L}$ double-distilled water and $2 \mu \mathrm{L}$ A, C, G or $\mathrm{T}$ extension mixture (Thermo sequenase primer cycle sequencing kit with 7-deaza-dGTP; Amersham Pharmacia Biotech). The sequencing primer was designed to hybridize to bases 75387558 of the bacteriophage $\lambda$-DNA (Figure $1 \mathrm{C}$ ). The capillary reactor was cycled through 20 temperature cycles, each consisting of the following steps: (i) $95^{\circ} \mathrm{C}$ for $30 \mathrm{~s}$; (ii) $68^{\circ} \mathrm{C}$ for $30 \mathrm{~s}$; and (iii) $72^{\circ} \mathrm{C}$ for $90 \mathrm{~s}$. The thermal cycling was accomplished by submerging the capillary reactor, which was capped on both ends with a quartz capillary plug (Micro-quartz, Phoenix, AZ, USA), in a temperature-controlled water bath. Following thermal cycling, the reactor was immediately heated to $95^{\circ} \mathrm{C}$ to remove extension products from the wallimmobilized sequencing template, and the reaction mixture from each capillary was pressure-injected into separate microcentrifuge tubes and subsequently loaded into the wells of the slab gel using a pipet (load volume of $1 \mu \mathrm{L}$ ).

The sequencing was performed on a LI-COR model 4000 automated nearIR DNA sequencer. The gel consisted of an $8 \%(\mathrm{w} / \mathrm{v})$ polyacrylamide gel (Long Ranger ${ }^{\mathrm{TM}}$ gel; BMA, Rockland, ME, USA) that used $7 \mathrm{M}$ urea as the denaturant and a $1 \times$ TBE buffer. The gel, $25 \mathrm{~cm}$ (width) $\times 41 \mathrm{~cm}$ (length), was run at $1650 \mathrm{~V}$ for $8 \mathrm{~h}$. Data acquisition and base-calling were performed using the LI-COR software on a PC.

To investigate the stability of the anchoring scaffold to typical thermalcycling conditions, several capillary microreactors were subjected to eight thermal cycles using the temperature program described above. The tethered template was subjected to 15 rounds of sequencing with each round analyzed on the LI-COR automated DNA sequencer. For these experiments, only a single terminator tract was analyzed (ddCTP).

\section{Fluorescence Dye Terminator Sequencing in Capillary Reactor}

A near-IR dye terminator was kindly prepared for us by Shaheer Kahn of PE-Applied Biosystems. The dye-labeled terminator was a sulfonated tri- carbocyanine dye that was tethered to a non-hydrogen bonding site of the nucleotide base using a propargyl amine linker (2). The capillary reactor was filled with the extension mixture, which consisted of $1 \mu \mathrm{L} 21$-bp sequencing primer $(1 \mu \mathrm{M}), 2 \mu \mathrm{L}$ reaction buffer, $1 \mu \mathrm{L}$ dNTP mixture, $1 \mu \mathrm{L}$ thermal sequenase enzyme and finally $1 \mu \mathrm{L}$ $(50 \mu \mathrm{M})$ of the dye-labeled terminator (ddGTP). The reaction mixture was pressure-injected into the capillary, which was sealed with the quartz plugs and subjected to the following thermal cycling conditions for 20 cycles: (i)

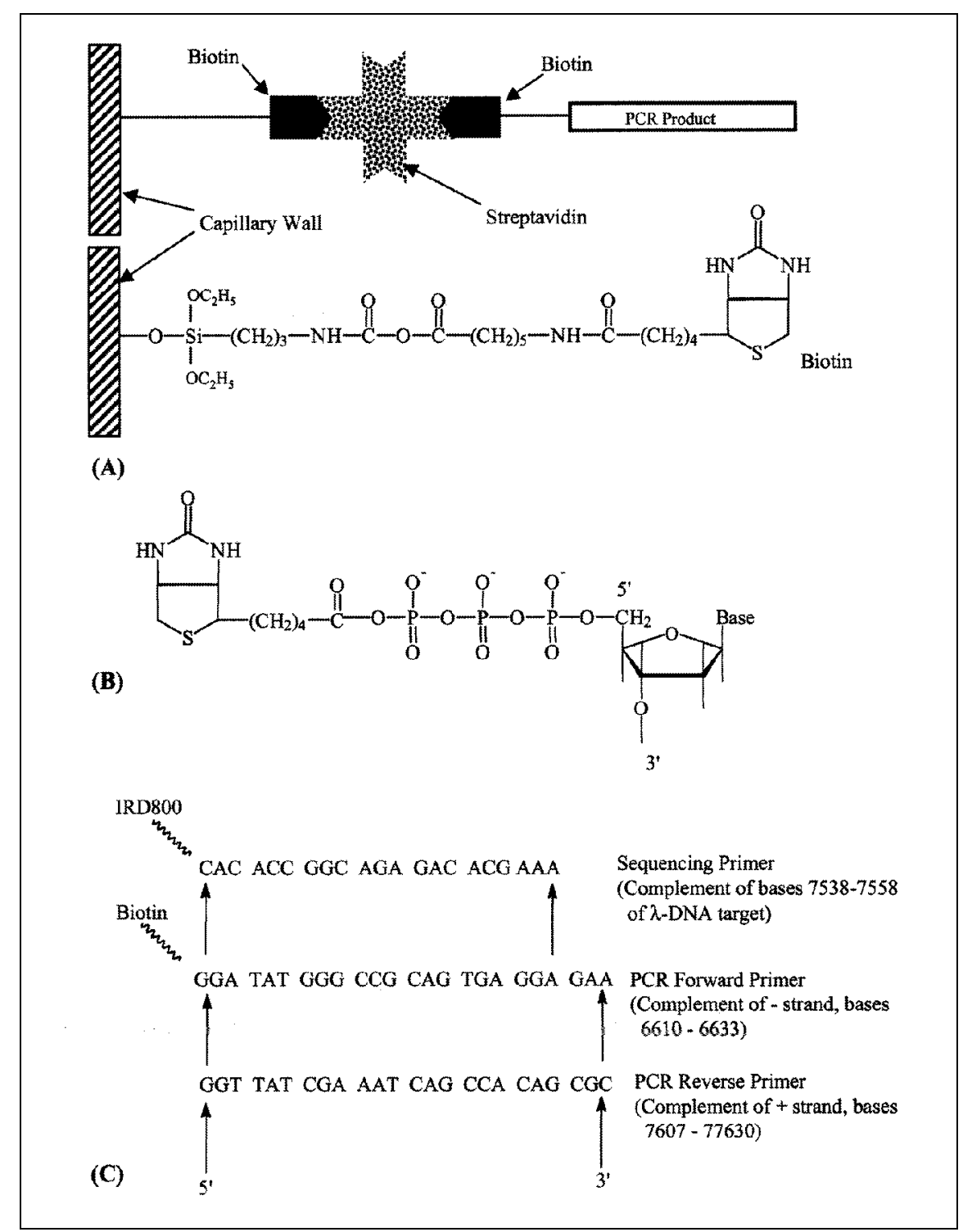

Figure 1. Chemical structures of immobilization layer, biotinylated linker to 5 ' end of oligonucleotide and sequence of PCR primers and sequencing primer. (A) Diagram of immobilization layer to fused silica capillary tube. (B) Sequences of the chemical linker of biotin to the PCR primer and (C) sequences of the PCR primers and sequencing primer with the dye label. 


\section{Short Technical Reports}

$95^{\circ} \mathrm{C}$ for $30 \mathrm{~s}$; and (ii) $60^{\circ} \mathrm{C}$ for $4 \mathrm{~min}$. Following thermal cycling, the contents of the capillary reactor were pressureinjected into a microcentrifuge tube and subjected to a cold ethanol precipitation to remove any excess dye-labeled ddGTP. After decanting the ethanol, the DNA was spun and dried in a centro-vap and finally taken up in 2 $\mu \mathrm{L}$ formamide loading buffer. One microliter of the solution was loaded onto an $8 \%(w / v)$ polyacrylamide slab gel with $7 \mathrm{M}$ urea as the denaturant. The electrophoresis and fluorescence detection were carried out on the LI-COR 4000 automated DNA sequencer.

\section{RESULTS AND DISCUSSION}

One important consideration to this reactor format is the amount of DNA template that is available for sequencing, which is determined by the amount of DNA immobilized to the wall of the capillary microreactor. In a previous publication, we determined that the amount of DNA immobilized is determined by the surface coverage of the streptavidin protein (16). In addition, even though three sites are potentially available for binding biotin on the wallanchored streptavidin, only one DNA molecule was tethered to a single streptavidin protein (16). With these considerations in mind, the calculated amount of DNA immobilized in this reactor (volume of $1.2 \mu \mathrm{L}$ ) was 3.9 pmol. By comparing the intensity of the resultant fluorescence signal of the ladder prepared in the capillary microreactor to that obtained from a ladder prepared in a conventional microcentrifuge tube containing a fixed amount of template DNA added to the reaction cocktail, the estimated amount of DNA attached to the wall of the capillary was about 1 pmol. This yielded an immobilization efficiency of approximately $25 \%$. We have attributed this disparity to inefficient anchoring of the biotin layer to the wall because of the sensitivity of the immobilization reactions to hydrolysis effects. It should be noted that the efficiency number measured this way could also be affected by steric considerations or inaccessibility of immobilized DNAs to sequencing reagents because the results were obtained using sequencing intensity data. However, we found that the level of DNA loading into the capillary microreactor was fairly consistent $( \pm 5 \%)$ from capillary to capillary when prepared in a similar fashion.

Because our automated DNA sequencer can process only one color, sequencing was carried out using the microcapillary solid-phase approach using single dye primer chemistry and four separate lanes of the gel. Here, the single base tracts prepared in each of four capillaries were loaded into the gel lanes of the sequencer. Figure 2 shows a trace of the sequencing data as well as the called bases. Using the base caller for this machine, we were able to read 589 bases, and the ambiguities from this trace were less than 10. Even after base 589, strong signal intensity was observed. In a simi-

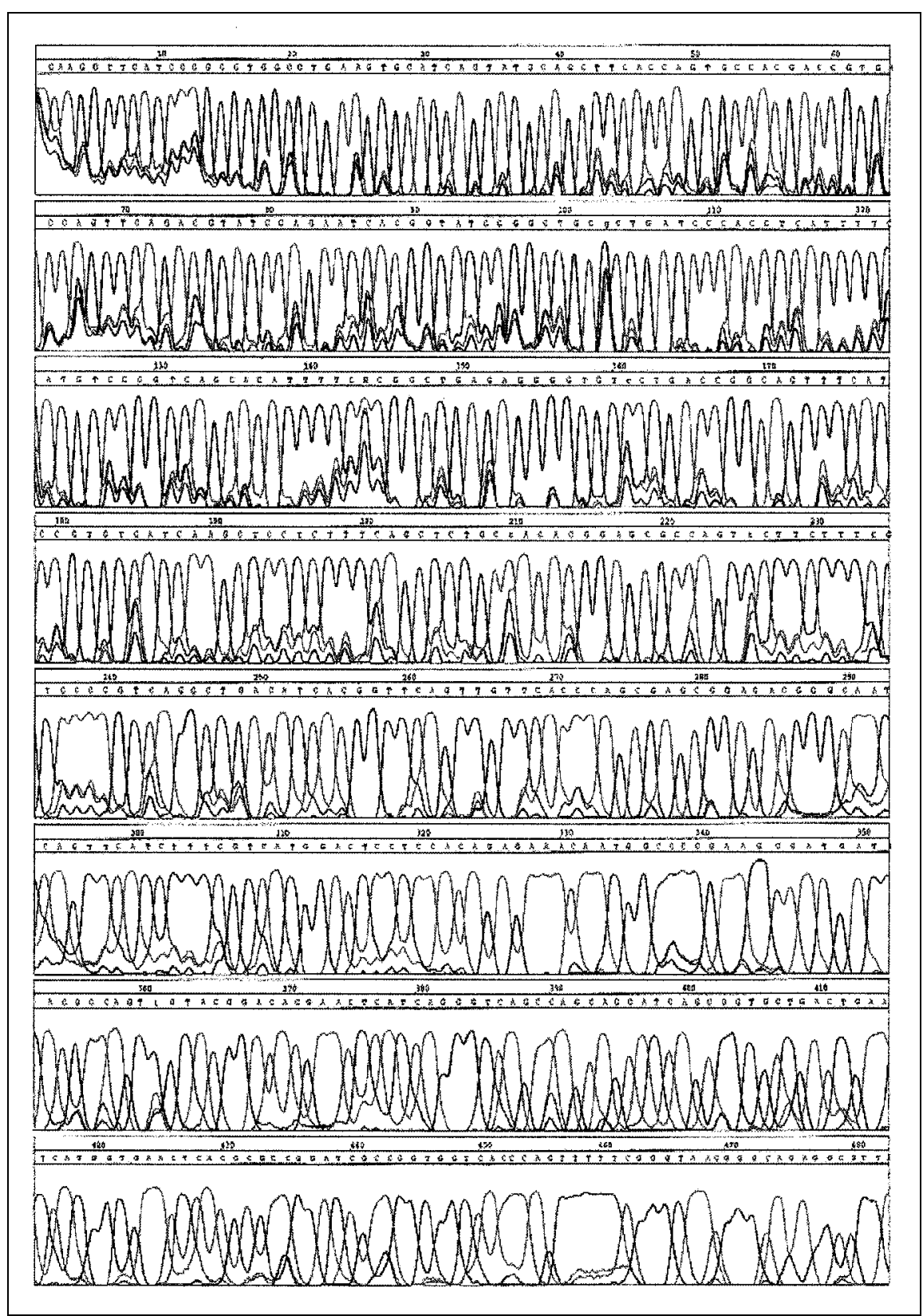

Figure 2. Sequencing trace (called bases 1-480) of a 1020-bp PCR-amplified $\lambda$-bacteriophage template immobilized to the wall of the capillary microreactor. Sequencing was performed using an IRD800 near-IR fluorescent label covalently attached to the custom-designed 21 -mer primer. In this sequencing run, a single dye was used with four sequencing lanes. Electrophoresis was performed on a LI-COR 4000 automated slab gel electrophoresis device with near-IR laser-induced fluorescence detection. 


\section{Short Technical Reports}

lar fashion, we generated sequence data on this same template using sequencing reactions prepared in conventional microcentrifuge tubes and a block thermal cycler. The results (data not shown) indicated a similar read length with the errors (in this case, defined as the number of ambiguities) equal to about 20 . Therefore, the capillary microreactor can produce high-quality sequencing data, which is comparable to conventional sample preparation formats. Improvements in read length could easily be achieved by improving electrophoresis conditions, through improvements in either the sieving gel or the electrophoresis operating parameters.

Our next set of experiments was focused on investigating the stability of the anchoring system to typical cycle sequencing conditions. For each round of sequencing, eight thermal cycles were performed on a single capillary solid-phase reactor as described in the
Materials and Methods section. After 15 sequencing rounds, the signal was clearly observable. The relative signal intensity for the same band selected from the trace was found to drop by only about $40 \%$ after 120 thermal cycles (15 rounds and eight thermal cycles/round). The impressive chemical stability of this tethering system arises from both the stability of the biotin:streptavidin couple and the ability of the siloxane bonds used to attach biotin to the wall of the capillary reactor to withstand the heating conditions required for cycle sequencing. In addition, we found that the streptavidin and immobilized template DNA can be stripped from the reactor by subjecting the capillary to heat $\left(>95^{\circ} \mathrm{C}\right)$ and $90 \%$ formamide (16). However, the wall-immobilized biotin is not removed from the capillary using these conditions because incubation with fresh streptavidin and the addition of biotinylated DNA will reactivate the capillary reactor.

One of the disadvantages of the dye primer chemistry used here is that the DNA polymerization must be done in four separate reactors and pooled before the electrophoresis, if four unique fluorescent probes can be used (four colors). Therefore, it would be particularly advantageous if dye terminator chemistry could be implemented, allowing the sequencing reactions to be performed in a single capillary microreactor. We evaluated the feasibility of using near-IR labeled terminators for this application. These experiments were carried out by accumulating data for a single tract $(\mathrm{G}-$ tract). The results produced a high-quality $G$ tract with the number of readable bases approaching 183 for this single base tract. In this case, we needed to remove excess dye terminator before gel loading using a cold ethanol precipitation since the free dye-terminator migrated at a rate equivalent to a 110-base 


\section{Short Technical Reports}

ssDNA fragment. This produced a large band in the electropherogram, which masked the signal from several bands in the DNA ladder. However, the need for an ethanol precipitation step could be alleviated if the extension products could remain captured on the wall of the capillary microreactor (only one thermal cycle). Simple pumping of clean buffer into the capillary before heat denaturation would allow effective removal of the excess dye-labeled ddNTP before gel loading.

\section{CONCLUSION}

We performed microsequencing in capillary tubes containing wall-immobilized DNA templates (solid-phase sequencing). Using a four-lane, single color dye primer sequencing run, a read length was obtained (589 bases) that was comparable to a conventional sample prep format. An advantage of this format is that the reactor can be scaled to ultra-small volumes, making it appropriate for loading sequencing samples directly to microseparation platforms, such as ultra-thin slab gels or capillary gels, and thus reducing the amount of reagents required for sample preparation. In addition, tethering the template to the wall of the microreactor would eliminate the loading of template onto the gel column, which has been shown to severely degrade the quality of sequencing data obtained in capillary gel electrophoresis (13). The advantages of traditional solid-phase sequencing are preserved in this format as well, such as producing high-quality, single-stranded templates and the ability to automate the sample preparation phases of the process. While we have been able to perform cycle sequencing in these microcapillaries, the use of a single thermal cycle will offer some potentially attractive features, especially when using dye terminator chemistry. For example, after a single thermal cycle, excess reagents, in particular dye-ddNTPs, can be removed from the sequencing products with no need for a cold ethanol precipitation. Another attractive feature of this solid-phase sequencing strategy is the potential to integrate the microreactor to capillary-based gel loaders. Recently, a pneumatic device was described that uses arrays of capillaries to transfer samples from titer plates to the wells of high-density gels to automate the loading process of slab gels (10). One can envision the use of a capillary containing immobilized single-stranded templates that would not only load the wells of the automated DNA sequencer but also prepare high-quality sequencing ladders, expanding on the existing capabilities of the capillary-based gel loader.

\section{REFERENCES}

1.Ansorge, W., B. Sproat, J. Stegemann and C. Schwager. 1986. A non-radioactive automated method for DNA sequence determination. J. Biochem. Biophys. Methods 13:315-323.

2.Flanagan, J.H., C. Owens, S. Romero, E. Waddell, S. Kahn, R. Hammer and S.A. Soper. 1998. Near-infrared heavy-atom-modified fluorescent dyes for base-calling in DNA-sequencing applications using temporal discrimination. Anal. Chem. 70:2676-2684

3.Hawkins, T.L., T. O'Connor-Morin, A. Roy and C. Santillan. 1994. DNA purification and isolation using a solid-phase. Nucleic Acids Res. 22:4543-4544.

4.Huang, S.-C., H. Swerdlow and K.D. Caldwell. 1994. Binding of biotinylated DNA to streptavidin-coated polystyrene latex. Anal. Biochem. 222:441-449.

5.Hultman, T., B. Staffan, T. Moks and M. Uhlén. 1991. Bidirectional solid-phase sequencing of in vitro-amplified plasmid DNA. BioTechniques 10:84-93.

6.Hultman, T., S. Stähl, E. Hornes and M. Uhlén. 1989. Direct solid phase sequencing of genomic and plasmid DNA using magnetic beads as solid support. Nucleic Acids Res. 17:4937-4947.

7.Hultman, T., S. Stähl, T. Moks and M. Uhlén. 1988. Approaches to solid phase DNA sequencing. Nucleosides Nucleotides 7:629-638.

8.McGinnis, M.D., M.P. Conrad, A.G.M. Bouwens, M.G.J. Tilanus and M.N. Kronick. 1995. Automated, solid-phase sequencing of DRB region genes using T7 sequencing chemistry and dye-labeled primers. Tissue Antigens 46:173-179.

9.Ohara, R., A. Tanaka and O. Ohara. 1997. Automated fluorescent DNA sequencing by a simplified solid-phase chemical sequencing method. BioTechniques 22:653-656.

10.Panussis, D.A., M.W. Cook, L.L. Rifkin, J.E. Snider, J.T. Strong, R.M. McGrane, R.K. Wilson and E.R. Mardis. 1998. A pneumatic device for the rapid loading of DNA sequencing gels. Genome Res. 8:543-548.

11.Prober, J.M., G.L. Trainor, R.J. Dam, F.W. Hobbs, C.W. Robertson, R.J. Zagursky, A.J. Cocuzza, M.A. Jensen and K. Baumeister. 1987. A system for rapid DNA sequencing with fluorescent chain-terminating dideoxynucleotides. Science 238:336-341.

12.Rolfs, A. and I. Weber. 1994. Fully-automated, nonradioactive solid-phase sequencing of genomic DNA obtained from PCR. BioTech- niques 17:782-787.

13.Ruiz-Martinez, M.C., O. Salas-Solano, E. Carrilho, L. Kotler and B.L. Karger. 1998. A sample purification method for rugged and high-performance DNA sequencing by capillary electrophoresis using replaceable polymer solutions. A. Development of the cleanup protocol. Anal. Chem. 70:1516-1527.

14.Schofield, J.P., D.S.C. Jones and M. Vaudin. 1993. Fluorescent and radioactive solid-phase dideoxy sequencing of polymerase chain reaction products in microtiter plates. Methods Enzymol. 218:93-103.

15.Smith, L.M., J.Z. Sander, R.J. Kaiser, P. Hughes, C. Dodd, C.R. Connell, C. Heiner, S.B.H. Kent and L.E. Hood. 1986. Fluorescence detection in automated DNA sequence analysis. Nature 321:674-678.

16.Soper, S.A., D.C. Williams, Y. Xu, S.J. Lassiter, Y. Zhang, S.M. Ford and R.C. Bruch. 1998. Sanger DNA-sequencing reactions performed in a solid-phase nanoreactor directly coupled to capillary gel electrophoresis. Anal. Chem. 70:4036-4043.

17.Stähl, S., T. Hultman, A. Olsson, T. Moks and M. Uhlén. 1988. Solid phase DNA sequencing using the biotin-avidin system. Nucleic Acids Res. 16:3025-3038.

18.Syvänen, A., T. Hultman, K. Aalto-Sertälä, H. Söderlund and M. Uhlén. 1991. Genetic analysis of the polymorphism of the human apolipoprotein E using automated solid-phase sequencing. GATA 8:117-123.

19.Tong, X. and L.M. Smith. 1992. Solid-phase method for the purification of DNA sequencing reactions. Anal. Chem. 64:2672-2677.

20.Uhlén, M. 1989. Magnetic separation of DNA. Nature 340:733-734.

21.Wahlberg, J., A. Holmberg, S. Bergh, T. Hultman and M. Uhlén. 1992. Automated magnetic preparation of DNA templates for solid phase sequencing. Electrophoresis 13:547-551.

22.Wang, S., M. Krinks and M. Moos. 1995. DNA sequencing from single phage plaques using solid-phase magnetic capture. BioTechniques 18:130-135.

The authors would like to thank the National Institutes of Health (National Human Genome Research Institute Grant No. HG01499) for financial support of this work. We would also like to thank Dr. Shaheer Kahn of PE Applied Biosystems for supplying us with the near-IR labeled terminator. Address correspondence to Professor Steven A. Soper, Department of Chemistry, Louisiana State University, Baton Rouge, LA 70803-1804, USA.Internet: steve.soper@chem.lsu.edu

Received 13 September 1999; accepted 4 February 2000.

\section{Yichuan Xu, Richard C. Bruch and Steven A. Soper Louisiana State University Baton Rouge, LA, USA}

